\title{
Lich-Gregoir vesico-ureteral reimplantation for duplex kidney anomalies in the pediatric population: a retrospective cohort study between laparoscopic and open surgery
}

\author{
Xiaojiang Zhu ${ }^{1 \#}$, Jun Wang ${ }^{1 \#}$, Haobo Zhu ${ }^{1 \#}$, Liqu Huang ${ }^{1}$, Chenjun Chen ${ }^{1}$, Lixia Wang ${ }^{1}$, Jun Dong ${ }^{1}$, \\ Zheng $\mathrm{Ge}^{1}$, Geng $\mathrm{Ma}^{1}$, Yunfei Guo ${ }^{1}$, Songming Huang ${ }^{2}$ \\ ${ }^{1}$ Department of Urology, Children's Hospital of Nanjing Medical University, Nanjing, China; ${ }^{2}$ Department of Nephrology, Children's Hospital of \\ Nanjing Medical University, Nanjing, China \\ Contributions: (I) Conception and design: X Zhu, J Wang, Y Guo, S Huang; (II) Administrative support: Y Guo, S Huang; (III) Provision of study \\ materials or patients: H Zhu, L Huang, J Dong; (IV) Collection and assembly of data: J Wang, Z Ge; (V) Data analysis and interpretation: X Zhu, C \\ Chen, G Ma; (VI) Manuscript writing: All authors; (VII) Final approval of manuscript: All authors. \\ \#These authors contributed equally to this work. \\ Correspondence to: Songming Huang. Department of Nephrology, Children's Hospital of Nanjing Medical University, Nanjing, China. Email: \\ chinachangzhou@126.com; Yunfei Guo. Department of Urology, Children’s Hospital of Nanjing Medical University, Nanjing, China. Email: \\ guoyunfei_nj@163.com.
}

Background: The aim of the study is to compare the Lich-Gregoir vesico-ureteral reimplantation in laparoscopy and open surgery.

Methods: In this case control study, we enrolled pediatric patients who were diagnosed with unilateral duplex kidney and had underwent surgical treatment. The surgical treatments were either conventional open surgery or laparoscopic surgery. We collected the basic demographic data and extracted the operativerelated statistics such as operation time, blood loss, length of hospital stay, pain level, and post-operative complications. The two groups were compared using Student's $t$-test.

Results: A total of 90 subjects were enrolled. Of the enrolled subjects, 35 underwent open surgery and 55 underwent laparoscopic surgery. There were no observable difference in the basic demographics between two groups $(\mathrm{P}>0.05)$. The duration of operation in laparoscopic surgery group was significantly shorter than in the open surgery group $(95.60 \pm 5.25$ vs. $108.70 \pm 3.12 \mathrm{~min}, \mathrm{P}=0.040)$. It was also noted that the amount of blood loss, length of hospital stay, drainage level, and the mean visual analog scale in laparoscopic group were significantly lower $(\mathrm{P}<0.05)$. The total incidence of complications in the laparoscopic and open surgery groups were $16.36 \%$ and $37.14 \%$, respectively.

Conclusions: Laparoscopic Lich-Gregoir vesico-ureteral reimplantation surgery management can be successful, clinically effective, and safe for pediatric population with functional duplex kidneys, and is better than the open surgery techniques.

Keywords: Lich-Gregoir procedures; laparoscopic; duplex kidney; vesico-ureteral reimplantation; pediatrics

Submitted Jun 17, 2020. Accepted for publication Nov 30, 2020.

doi: $10.21037 /$ tp-20-163

View this article at: http://dx.doi.org/10.21037/tp-20-163

\section{Introduction}

Duplex kidney is a common congenital abnormality of the urinary system in the pediatric population. Its incidence is $1 \%$ worldwide $(1,2)$. Most cases of duplex kidneys become apparent in childhood with classic symptoms that signify the disease. Such symptoms include incontinence, recurrent urinary tract infections (UTIs) and ureteropelvic junction obstruction $(3,4)$. For patients with duplex kidneys 
and ectopic opening of ureter complicated by leakage, obstruction and/or severe vesico-ureteral reflux (VUR), vesico-ureteral reimplantation is considered to be the optimal therapy instead of heminephrectomy (5).

For a long time, open surgery was considered as the conventional surgical treatment for pediatric patients with duplex kidney. But in recent years, laparoscopy has been gaining significant acceptance in pediatric urology $(6,7)$. Minimally invasive procedures such as laparoscopy and endoscopic injection have demonstrated relatively equivalent short-term and long-term outcomes with reduced complications and rapid rehabilitation in both adult and pediatric patients when compared to open surgery $(8,9)$. With the incorporation of new technical advances, the laparoscopic vesico-ureteral reimplantation has been gradually incorporated into the management of patients with duplex kidney abnormalities. Conventional vesico-ureteral reimplantation can be divided into three categories: extra-vesical, intravesical and union of Intra- and extra-vesical approaches (10). Among various intra-vesical approaches, Cohen and PolitanoLeadbetter methods are the most widely accepted and applied in clinical practice $(11,12)$. However, Lich-Gregoir procedure is recommended for ureteral obstruction, VUR and ectopic opening of ureter treatment because it causes less damage to patients, is associated with handy skills, and guarantees shorter hospital stays, $(13,14)$. Our treatment center relies on both open surgery and laparoscopic surgery to treat pediatric duplex kidney using the Lich-Gregoir procedure. This is due to the easier access granted by the open Lich-Gregoir vesicoureteral reimplantation for younger surgeons as well as the higher success rate of open surgery in previous reports. Since the minimal surgical techniques have attractive advantages for pediatric patients, it was expedient to compare open and laparoscopic surgery using the Lich-Gregoir vesico-ureteral reimplantation in pediatric patients in our center.

Therefore, the aim of this study is to retrospectively investigate the clinical efficacy and safety of laparoscopic Lich-Gregoir vesico-ureteral reimplantation on pediatric patients diagnosed with duplex kidneys when compared to conventional open surgery. We present the following article in accordance with the STROBE reporting checklist (available at http://dx.doi.org/10.21037/tp-20-162).

\section{Methods}

\section{Study population}

This study was conducted as a retrospective 6-month study and case-control study. We considered pediatric patients admitted into our center (Department of Urology, The Affiliated Children's Hospital of Nanjing Medical University) from Jan $1^{\text {st }}, 2015$ to Jan $1^{\text {st }}, 2018$ for assessment. Our study has been reported in line with the STROCSS criteria (15). We presented the assessment criteria as follows: (I) patients had to be between 6 months and 18 years of age at the time of surgery; (II) patients had to be diagnosed with unilateral duplex kidney using Doppler B ultrasound, intravenous pyelography, abdominal computer tomography (CT), magnetic resonance urography (MRU) or Retrograde cystoscopy; (III) all duplex kidneys had to be confirmed to be functional using retrograde cystoscopy; (IV) patients' legal guardians had to be willing to participate in the study.

\section{Data extraction}

Outpatient and inpatient records for each individual patient in our study were extracted and reviewed by two independent authors (XJ Zhu and J Wang). Any disagreements were resolved either by discussion or by inviting a third reviewer (YF Guo). Basic demographic data analyzed included gender, age, weight, side of duplex kidneys, renal function, complications of duplex kidneys, radiological images (ureters and bladder ultrasonography, intravenous pyelography, CT and MRU), operative records (operative time, hospital stay, blood loss) and post-operative complications.

\section{Surgical techniques}

Laparoscopic Lich-Gregoir vesico-ureteral reimplantation surgery was performed with tracheal intubation with aspiration. All patients underwent cystoscopy with the ureteral intubation before laparoscopic surgery. The patients were placed in the supine position and put under general anesthesia. Pneumoperitoneum was created with a Veress needle and $5 \mathrm{~mm}$ Trocars were placed in the manipura, the right and left margin of rectus abdominis respectively. The distal portion of duplex ureter was dissected and clipped. The section of overdistension or stricture in the proximal ureter was resected to create an appropriate ureter tube. The myometrium and mucous layer of rear bladder side wall was cut to a $1 \mathrm{~cm}$ size. Subsequently, the cut ureteral opening was anastomosed to the bladder mucosa, and the double-J stent was placed before closing the anastomosis. The double-J stent was then removed by cystoscopy after $1-1.5$ months post-surgery. 
Open surgery was performed in line with the conventional procedures $(16,17)$. A Pfannenstiel incision was made and flanked to the outer edge of the rectus abdominis to the aponeurosis of the external oblique muscle. The ureter was defined at iliac vein level and dissection was continued through ureterovesical junction until the stenosis section was identified. The strictured ureteral segment was clipped and the proximal section was spatulated. A submucosal tunnel was created and the mucosa detached from the distal end of tunnel. Then, a double-J stent was placed before performing the final suture. Drainage strips were removed after 1-2 days following the operation and the catheter was removed after 7-9 days.

\section{Outcomes}

The primary outcome for consideration in this study was the operation time, while the intraoperative and perioperative variables, including blood loss, the length of hospital stay, drainage level, and visual analog scale (VAS) score, were the secondary outcomes. In addition, the postoperative complications were also included in the secondary outcomes. Evaluation was performed to assess severity of pain declared by the patient. In the interpretation of the VAS scores, 10 represented maximum pain and 0 represented no pain (18).

\section{Statistical analysis}

The continuous variables were presented as mean \pm standard deviation (SD). The Student's $t$-test was used for the comparison of the mean age and operation-related statistics and a $\mathrm{P}$ value less than 0.05 was considered to be statistically significant. The statistical analysis was done using the SPSS software (Version16.0, IBM Corp., Armonk, NY, USA).

\section{Ethical Statement}

The study protocol was in accordance with the ethical standards of the Declarations of Helsinki and Istanbul as revised in 2013. This research was approved by the local Ethics Committee of Nanjing Medical University Affiliated Nanjing Children's Hospital (No. 201801172-1). Written informed consent was obtained from all parents of included children.

\section{Results}

We enrolled a total of 90 pediatric patients in our study after performing the eligibility assessment in accordance with the inclusion criteria. The case numbers of open surgery and laparoscopic groups were 35 and 55 respectively. The baseline patient demographic data of patients enrolled were as shown in Table 1. There was no observable significant difference in the basic characteristics of participants between the two groups.

All the procedures were completed without intraoperative complications and no conversion to open surgery in the laparoscopic group was performed. Operative time was significantly shorter statistically during laparoscopic surgery compared to open surgery $(95.60 \pm 5.25$ vs. $108.70 \pm 3.12 \mathrm{~min}, \mathrm{P}=0.040$; Table 2). When compared with the open surgery group, the amount of blood loss in laparoscopic group was significantly reduced and the length of hospital stay was also shortened (Blood loss: 11.40 \pm 1.14 vs. $44.10 \pm 4.112 \mathrm{~mL}, \mathrm{P}<0.0001$; Hospital stay: $2.30 \pm 0.34$ vs. $7.50 \pm 0.60$ days, $\mathrm{P}<0.0001$; Table 2). Moreover, the drainage level was significantly decreased with the use of laparoscopic surgery $(15.10 \pm 1.82$ vs. $23.70 \pm 1.45 \mathrm{~mL}, \mathrm{P}=0.0016$; Table 2). At six hours postoperation, the mean VAS score was $4.69 \pm 0.52$ in laparoscopic group and $6.63 \pm 0.46$ in open surgery group $(\mathrm{P}=0.0089)$. Afterward, the mean VAS score at post-operative day 1 was $3.38 \pm 0.44$ in laparoscopic group. This was significantly lower than in open surgery group ( $3.38 \pm 0.44$ vs. $4.25 \pm 0.41, \mathrm{P}=0.0016$; Table 2 ).

Postoperative complications were as summarized in Table 3. For the two groups, the total incidences of complications were $16.36 \%$ in laparoscopic group and $37.14 \%$ in open group $(\mathrm{P}=0.025)$. Most complications resolved spontaneously or after medical therapy without the need to perform a new surgical procedure. One exception for this was one case in the open surgery group where an intervention was required to correct urinary retention.

\section{Discussion}

Both laparoscopic and open surgery methods of heminephrourterectomy are well-recognized for the treatment of duplex kidney anomalies in the pediatric population (19). However, we attempted to combine the Lich-Gregoir vesico-ureteral reimplantation surgery with the laparoscopic techniques for functional duplex kidneys. It gave us a chance to explore the clinical efficacy and safety of the procedure. In this study, we found out that using the laparoscopic surgery and the Lich-Gregoir vesico-ureteral reimplantation surgery method provided favorable and substantial value to the pediatric patients with functional 
Table 1 Basic demographics of eligible pediatric patients in this study

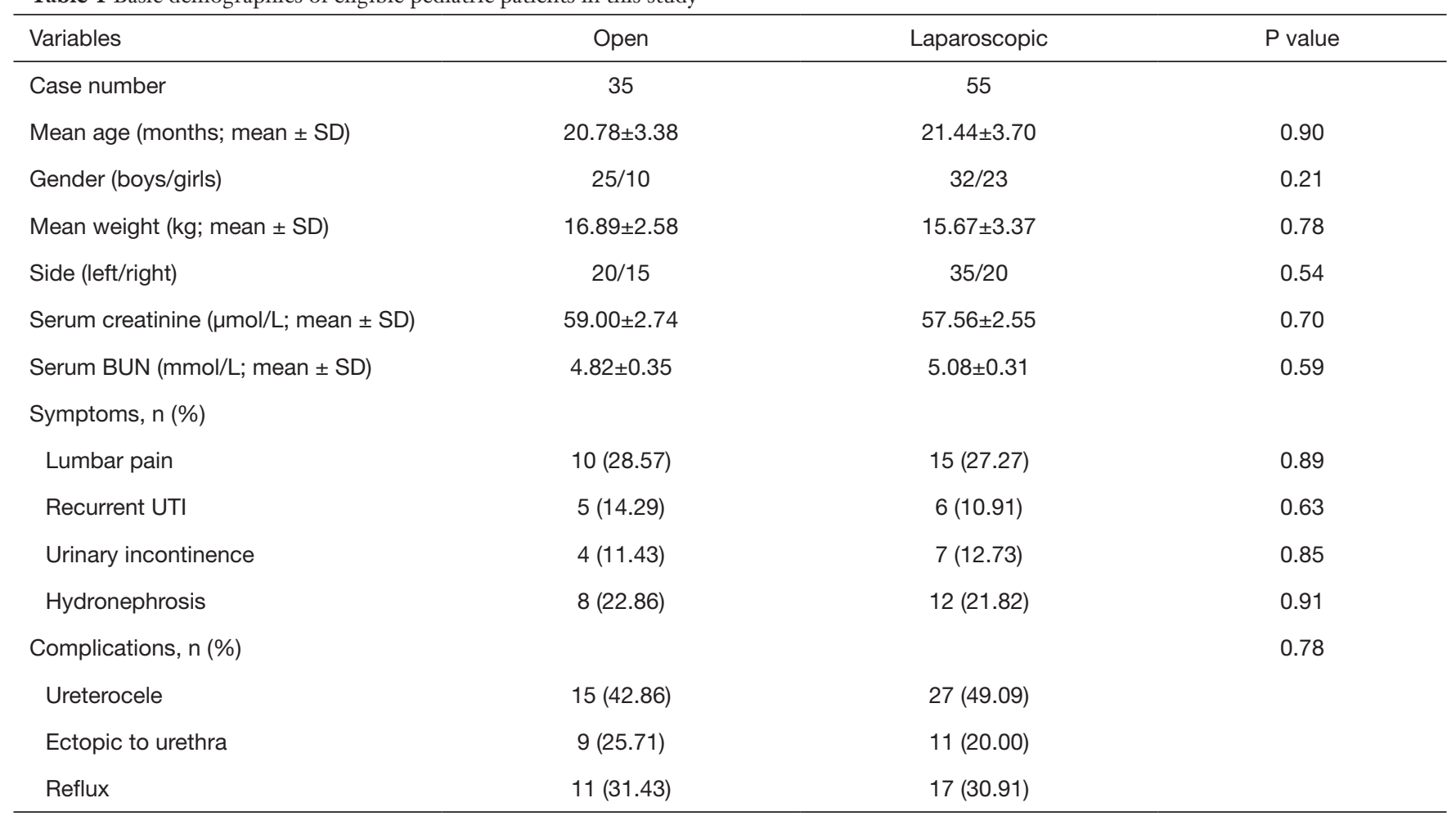

SD, standard deviations; BUN, blood urine nitrogen; UTI, urinary tract infection.

Table 2 Outcomes of Lich-Gregoir vesico-ureteral reimplantation surgery in open and laparoscopic groups

\begin{tabular}{lccc}
\hline Variables & Open & Laparoscopic & P value \\
\hline Operation time (min; mean \pm SD) & $108.70 \pm 3.12$ & $95.60 \pm 5.25$ & 0.040 \\
Blood loss (mL; mean \pm SD) & $44.10 \pm 4.11$ & $11.40 \pm 1.14$ & $<0.0001$ \\
Hospital stay (days; mean \pm SD) & $7.50 \pm 0.60$ & $2.30 \pm 0.34$ & $<0.0001$ \\
Drainage level (mL; mean \pm SD) & $23.70 \pm 1.45$ & $15.10 \pm 1.82$ & 0.0016 \\
VAS Day 0 (mean \pm SD) & $6.63 \pm 0.46$ & $4.69 \pm 0.52$ & 0.0089 \\
VAS Day 1 (mean \pm SD) & $4.25 \pm 0.41$ & $3.38 \pm 0.44$ & 0.0016 \\
\hline
\end{tabular}

$\mathrm{SD}$, standard deviations; VAS, visual analog scale.

duplex kidneys, except for longer operative time. No significant complications were observed when compared to the conventional open surgery.

Open surgical procedures have good long-term outcomes with a reported success rate of more than $80 \%$ (20). Nevertheless, longer hospital stays, extra blood loss and additional pain management are the common downsides of this procedure. This does not provide convenient outcomes for the pediatric population (16) hence, the need for minimally invasive techniques, such as laparoscopic procedures in pediatric urology. In this study, we also made significant findings in the similar clinical outcomes after comparing open and laparoscopic surgeries. These included longer hospital stays, higher amount of blood loss, extra drainage level and additional pain. These findings were consistent with previous reports, suggesting laparoscopic Lich-Gregoir vesico-ureteral reimplantation procedures are preferable for the treatment of functional duplex kidneys. As Ehrlich et al. (21) reported, the first case of laparoscopic Lich-Gregoir surgery for the treatment of pediatric VUR 
Table 3 Postoperative complications in open and laparoscopic group

\begin{tabular}{lcc}
\hline Variables & Open $(\mathrm{n}=35)$ & Laparoscopic $(\mathrm{n}=55)$ \\
\hline All complications & $13(37.14)$ & $9(16.36)$ \\
Fever & $2(5.71)$ & $1(1.82)$ \\
UTIs & $3(8.57)$ & $2(3.64)$ \\
Bleeding & $1(2.86)$ & $1(1.82)$ \\
Urinary retention & $1(2.86)$ & $0(0)$ \\
Nausea/vomiting & $2(5.71)$ & $2(3.64)$ \\
Reoperation & $1(2.86)$ & $0(0)$ \\
Persistent hematuria & $1(2.86)$ & $1(1.82)$ \\
Reflux persistence & $2(5.71)$ & $2(3.64)$ \\
\hline
\end{tabular}

UTIs, urinary tract infections.

was performed in 1994. But there has been an increase in the application of the laparoscopic Lich-Gregoir procedures in recent years. During our single-center experience, we noticed that the laparoscopic Lich-Gregoir procedures were highly indicated for the VUR cases with Grade III or IV, as well as for the Grade V VUR cases without distal ureteral distortion or over-distension. Additionally, we routinely performed the cystoscopy with the ureteral intubation for patients with ectopic ureter opening to distinguish the normal from duplex ureter during laparoscopic LichGregoir procedures.

It was also found that longer operative time in laparoscopic Lich-Gregoir procedures can be associated with several factors. First, as mentioned above, a cystoscopy for the incubation of ureteral catheter was routinely performed to distinguish the normal and duplex ureter. This inevitably prolonged the operative time of laparoscopic surgery. Then, the ureteral suture tended to a difficult and time-consuming process during the procedures. Besides, the adnexa uteri always affected the exposure of surgical visual field in the process of laparoscopic Lich-Gregoir procedures for girls. Dissection of adnexa uteri would be performed, contributing to the additional operative time. Although the operative time was significantly longer in laparoscopic procedures, the extra operative time was still acceptable regarding the pronounced advantages. The advantages included smaller bleeding amount, lower post-operative pain levels, and shorter hospital stays.

Rate of complications reported for laparoscopic surgery in our study was favorable and significant. Most of the complications were mild to moderate and included fever,
UTIs and gastroenteric reactions. One of the most common complications observed after reconstructive ureteric surgery is recurrent stenosis. It has an incidence of approximately $5 \%$ (22). In our study, we reported 2 cases $(5.71 \%)$ of urinary reflux in open surgery group and 2 cases (3.64\%) in laparoscopic group, owing to the post-operative ureteral stenosis which was consistent with the reported statistics. Therefore, our study indicated that the laparoscopic LichGregoir procedure for vesico-ureteral reimplantation is evidently safe for pediatric patients.

We noticed some limitations in our study. First, this was a retrospective study without more evidential design. Our follow-up duration for this study was 6 months, a relatively short-term duration to compare open surgery and laparoscopic groups. The long-term outcomes should still be followed to confirm the efficacy and safety of the procedures.

In conclusion, the management of duplex kidney anomalies using laparoscopic Lich-Gregoir vesico-ureteral reimplantation surgery can be successful, clinically effective and safe for pediatric population and the procedure is way more advantageous than open surgery techniques.

\section{Acknowledgments}

Funding: None.

\section{Footnote}

Reporting Checklist: The authors have completed the STROBE reporting checklist. Available at http://dx.doi. 
org/10.21037/tp-20-162

Data Sharing Statement: Available at http://dx.doi. org/10.21037/tp-20-162

Conflicts of Interest: All authors have completed the ICMJE uniform disclosure form (available at: http://dx.doi. org/10.21037/tp-20-162). The authors have no conflicts of interest to declare.

Ethical Statement: The authors are accountable for all aspects of the work in ensuring that questions related to the accuracy or integrity of any part of the work are appropriately investigated and resolved. The study protocol was in accordance with the ethical standards of the Declarations of Helsinki and Istanbul as revised in 2013. This research was approved by the local Ethics Committee of Nanjing Medical University Affiliated Nanjing Children's Hospital (No. 201801172-1). Written informed consent was obtained from all parents of included children.

Open Access Statement: This is an Open Access article distributed in accordance with the Creative Commons Attribution-NonCommercial-NoDerivs 4.0 International License (CC BY-NC-ND 4.0), which permits the noncommercial replication and distribution of the article with the strict proviso that no changes or edits are made and the original work is properly cited (including links to both the formal publication through the relevant DOI and the license). See: https://creativecommons.org/licenses/by-nc-nd/4.0/.

\section{References}

1. Privett JT, Jeans WD, Roylance J. The incidence and importance of renal duplication. Clin Radiol 1976;27:521-30.

2. Hunziker M, Kutasy B, D'Asta F, et al. Urinary tract anomalies associated with high grade primary vesicoureteral reflux. Pediatr Surg Int 2012;28:201-4.

3. Whitten SM, Wilcox DT. Duplex systems. Prenat Diagn 2001;21:952-7.

4. Malek RS, Kelalis PP, Stickler GB, et al. Observations on ureteral ectopy in children. J Urol 1972;107:308-13.

5. Malik RD, Pariser JJ, Gundeti MS. Outcomes in Pediatric Robot-Assisted Laparoscopic Heminephrectomy Compared with Contemporary Open and Laparoscopic Series. J Endourol 2015;29:1346-52.

6. Smaldone MC, Sweeney DD, Ost MC, et al. Laparoscopy in paediatric urology: present status. BJU Int 2007;100:143-50.

7. Seibold J, Janetschek G, Bartsch G. Laparoscopic surgery in pediatric urology. Eur Urol 1996;30:394-9.

8. Babu R, Chandrasekharam VVS. A systematic review and meta-analysis comparing outcomes of laparoscopic extravesical versus trans vesicoscopic ureteric reimplantation. J Pediatr Urol 2020;16:783-9.

9. Chandrasekharam VVS, Babu R. Robot-assisted laparoscopic extravesical versus conventional laparoscopic extravesical ureteric reimplantation for pediatric primary vesicoureteric reflux: a systematic review and metaanalysis. Pediatr Surg Int 2020;36:1371-8.

10. Zhong $\mathrm{W}$, Yao L, Cui H, et al. Laparoscopic ureteral reimplantation with extracorporeal tailoring and direct nipple ureteroneocystostomy for adult obstructive megaureter: long-term outcomes and comparison to open procedure. Int Urol Nephrol 2017;49:1973-8.

11. Nabavizadeh B, Keihani S, Hosseini Sharifi SH, et al. Insertion of a single double-J stent for bilateral open ureteral reimplantation: introducing a novel technique and assessment of feasibility. Int Urol Nephrol 2016;48:1015-9.

12. Soh S, Kobori Y, Shin T, et al. Transvesicoscopic ureteral reimplantation: Politano-Leadbetter versus Cohen technique. Int J Urol 2015;22:394-9.

13. Lopez M, Melo C, Francois M, et al. Laparoscopic extravesical transperitoneal approach following the lichgregoir procedure in refluxing duplicated collecting systems: initial experience. J Laparoendosc Adv Surg Tech A 2011;21:165-9.

14. Lapointe SP, Barrieras D, Leblanc B, et al. Modified LichGregoir ureteral reimplantation: experience of a Canadian center. J Urol 1998;159:1662-4.

15. Agha RA, Borrelli MR, Vella-Baldacchino M, et al. The STROCSS statement: Strengthening the Reporting of Cohort Studies in Surgery. Int J Surg 2017;46:198-202.

16. Rassweiler JJ, Gozen AS, Erdogru T, et al. Ureteral reimplantation for management of ureteral strictures: a retrospective comparison of laparoscopic and open techniques. Eur Urol 2007;51:512-22; discussion 522-3.

17. Nourizadeh D, Houshagi A, Goldust M. Lich-gregoir procedure in treatment of the vesicoureteral reflux. Pak J Biol Sci 2013;16:426-30.

18. Reed MD, Van Nostran W. Assessing pain intensity with the visual analog scale: a plea for uniformity. J Clin Pharmacol 2014;54:241-4.

19. Seibold J, Schilling D, Nagele U, et al. Laparoscopic 
heminephroureterectomy for duplex kidney anomalies in the pediatric population. J Pediatr Urol 2008;4:345-7.

20. Sakellariou P, Protopapas AG, Voulgaris Z, et al. Management of ureteric injuries during gynecological operations: 10 years experience. Eur J Obstet Gynecol Reprod Biol 2002;101:179-84.

21. Ehrlich RM, Gershman A, Fuchs G. Laparoscopic

Cite this article as: Zhu X, Wang J, Zhu H, Huang L, Chen C, Wang L, Dong J, Ge Z, Ma G, Guo Y, Huang S. Lich-Gregoir vesico-ureteral reimplantation for duplex kidney anomalies in the pediatric population: a retrospective cohort study between laparoscopic and open surgery. Transl Pediatr 2021;10(1):26-32. doi: $10.21037 / \mathrm{tp}-20-163$ vesicoureteroplasty in children: initial case reports. Urology 1994;43:255-61.

22. Lim SK, Shin TY, Rha KH. Current status of robot assisted laparoscopic radical nephroureterectomy for management of upper tract urothelial carcinoma. Curr Urol Rep 2013;14:138-46. 


\section{Supplementary}

Table S1 Demographic and other baseline information of the children with CHD

\begin{tabular}{lccc}
\hline Indicator & $\mathrm{N}($ mean $\pm \mathrm{SD})$ & Normal blood coagulation & Abnormal blood coagulation \\
\hline Sex $(\mathrm{M} / \mathrm{F})$ & $1,690(941 / 749)$ & $497(50.35 \%) / 490(49.65 \%)$ & $444(63.16 \%) / 259(36.84 \%)$ \\
Age & $1,690(1.751 \pm 2.731)$ & $987(2.555 \pm 3.132)$ & $703(0.623 \pm 1.405)$ \\
PT & $471(11.221 \pm 0.664)$ & $415(11.110 \pm 0.658)$ & $56(11.202 \pm 0.709)$ \\
APTT & $471(34.655 \pm 2.805)$ & $415(34.692 \pm 2.790)$ & $56(34.382 \pm 2.927)$ \\
INR & $471(0.974 \pm 0.056)$ & $415(0.973 \pm 0.056)$ & $56(0.983 \pm 0.063)$ \\
FBG & $471(2.497 \pm 0.408)$ & $415(2.499 \pm 0.402)$ & $56(2.480 \pm 0.457)$ \\
WBC & $1,378(10.291 \pm 5.107)$ & $845(9.643 \pm 4.732)$ & $533(11.318 \pm 5.501)$ \\
PLT & $1,109(328.318 \pm 145.780)$ & $678(324.153 \pm 133.130)$ & $431(334.870 \pm 163.663)$ \\
HB & $745(120.728 \pm 27.941)$ & $478(119.839 \pm 22.941)$ & $267(122.318 \pm 35.154)$ \\
HCT & $745(36.771 \pm 7.886)$ & $478(36.424 \pm 6.345)$ & $267(37.394 \pm 10.058)$ \\
MCV & $1,378(82.990 \pm 8.884)$ & $845(81.277 \pm 8.063)$ & $533(85.708 \pm 9.439)$ \\
MCHC & $1,378(333.340 \pm 18.799)$ & $845(335.298 \pm 18.706)$ & $543(27.256 \pm 3.054)$ \\
MCH & $1,378(27.690 \pm 3.381)$ & $845.236 \pm 18.544)$ & $533(28.377 \pm 3.743)$
\end{tabular}

PT, prothrombin time; APTT, activated partial thromboplastin time; INR, international standardized ratio; FBG, fibrinogen; WBC, white blood cell count; PLT, platelet count; HB, hemoglobin; HCT, hematocrit; MCV, mean corpuscular volume; MCHC, mean corpuscular hemoglobin concentration; $\mathrm{MCH}$, mean corpuscular hemoglobin.

Table S2 Parameters of conventional coagulation tests and their reference range

\begin{tabular}{lccc}
\hline Parameter & Normal & Hypocoagulation & Hypercoagulation/hyperfibrinolysis \\
\hline PT (s)/INR & $9.4-12.5$ & $>12.5$ & $<9.4$ \\
INR & $0.8-1.2$ & $>1.2$ & $<0.8$ \\
APTT (s) & $25.1-38.4$ & $>38.4$ & $<25.1$ \\
Fib (g/L) & $2-4$ & $<2$ & $>4$ \\
D-dimer (mg/L) & $0-0.24$ & - & $>0.24$ \\
ATIII (\%) & $83-128$ & $>128$ & $<83$ \\
\hline
\end{tabular}

PT, prothrombin time; INR, international standardized ratio; APTT, activated partial thromboplastin time; ATIII, anticoagulant enzyme III. 